\title{
Correction: Mutations in the DNA methylation pathway and number of driver mutations predict response to azacitidine in myelodysplastic syndromes
}

\author{
M. Teresa Cedena ${ }^{1,2, *}$, Inmaculada Rapado ${ }^{1,2, *}$, Alejandro Santos-Lozano ${ }^{2,3}$, Rosa \\ Ayala1,2, Esther Onecha ${ }^{1,2}$ María Abaigar ${ }^{4}$, Esperanza Such ${ }^{5}$, Fernando Ramos $^{6}$, \\ José Cervera ${ }^{5,7}$, María Díez-Campelo ${ }^{4,8}$, Guillermo Sanz ${ }^{5}$, Jesús Hernández Rivas ${ }^{4,8}$, \\ Alejandro Lucía ${ }^{2,9}$ and Joaquin Martínez-López ${ }^{1,2}$ \\ ${ }^{1}$ Hematology Department, Hospital Universitario 12 Octubre, CNIO, Universidad Complutense, Madrid, Spain \\ 2 Research Institute of Hospital 12 de Octubre ('i+12'), Madrid, Spain \\ ${ }^{3}$ GIDFYS, European University Miguel de Cervantes, Valladolid, Spain \\ ${ }^{4}$ IBSAL, Cancer Research Center (USAL-CSIC), Salamanca, Spain \\ ${ }^{5}$ Hematology Department, Hospital Universitario La Fe, Valencia, Spain \\ ${ }^{6}$ Hematology Department, Hospital Universitario de León, and IBIOMED, Universidad León, León, Spain \\ ${ }^{7}$ Genetics Unit, Hospital Universitario La Fe, Valencia, Spain \\ ${ }^{8}$ Hematology Department, Hospital Universitario de Salamanca, Salamanca, Spain \\ ${ }^{9}$ Universidad Europea de Madrid, Madrid, Spain \\ * These authors have contributed equally to this work \\ Published: July 20, 2018
}

Copyright: Cedena et al. This is an open-access article distributed under the terms of the Creative Commons Attribution License 3.0 (CC BY 3.0), which permits unrestricted use, distribution, and reproduction in any medium, provided the original author and source are credited.

This article has been corrected: In the Materials and Methods section, the data access link is incorrect. In addition, reference 37 was also listed incorrectly. The proper information is given below:

Materials and Methods: The data discussed in this publication have been deposited in the NCBI Sequence Read Archive (SRA) [37] and are accessible through (http://www.ncbi.nlm.nih.gov/sra/SRP102906).

References: 37. Leinonen R, Sugawara H, Shumway M. The sequence read archive. Nucleic Acids Res. 2011; 39 (Database issue): D19-D21.

Original article: Oncotarget. 2017; 8:106948-106961. https://doi.org/10.18632/oncotarget.22157 